\title{
Comparing State-Space Multivariable Controls to Multi- SISO Controls for Load Reduction of Drivetrain-Coupled Modes on Wind Turbines through Field-Testing
}

\author{
Paul A. Fleming ${ }^{1}$ \\ National Wind Technology Center, National Renewable Energy Laboratory, Golden, Colorado \\ Jan-Willem van Wingerden ${ }^{2}$ \\ Delft University of Technology, Delft, The Netherlands \\ and \\ Alan D. Wright ${ }^{3}$ \\ National Wind Technology Center, National Renewable Energy Laboratory, Golden, Colorado
}

\begin{abstract}
In this paper we present results from an ongoing controller comparison study at the National Renewable Energy Laboratory's (NREL's) National Wind Technology Center (NWTC). The intention of the study is to demonstrate the advantage of using modern multivariable methods for designing control systems for wind turbines versus conventional approaches. We will demonstrate the advantages through field-test results from experimental turbines located at the NWTC. At least two controllers are being developed sideby-side to meet an incrementally increasing number of turbine load-reduction objectives. The first, a multiple single-input, single-output (m-SISO) approach, uses separately developed decoupled and classicially tuned controllers, which is, to the best of our knowledge, common practice in the wind industry. The remaining controllers are developed using state-space multiple-input and multiple-output (MIMO) techniques to explicity account for coupling between loops and to optimize given known frequency structures of the turbine and disturbance.

In this first publication from the study, we present the structure of the ongoing controller comparison experiment, the design process for the two controllers compared in this phase, and initial comparison results obtained in field-testing.
\end{abstract}

\section{Nomenclature}

CART3 Controls Advanced Research Turbine (3-Bladed)

$\mathrm{H}_{\infty} \quad \mathrm{H}$-inifinity, a control design approach for synthesizing robust controllers

HSS High-speed shaft

m-SISO multiple SISO loops

MIMO Multiple-input multiple-outout

NREL National Renewable Energy Laboratory

NWTC National Wind Technology Center

PI Proportional-integral

SIMO Single-input multiple-output

SISO Single-input single-output

SS side-to-side

\footnotetext{
${ }^{1}$ Research Engineer, National Wind Technology Center (NWTC), National Renewable Energy Laboratory (NREL), 1617 Cole Blvd., Mailstop 3811, AIAA member.

${ }^{2}$ Assistant Professor, Delft Center for Systems and Control, 2628 CD, Delft, AIAA member

${ }^{3}$ Senior Engineer, National Wind Technology Center (NWTC), National Renewable Energy Laboratory (NREL), 1617 Cole Blvd., Mailstop 3811, AIAA member.
} 


\section{Introduction}

$\mathrm{T}$

HIS article presents the results of a controller comparison study for wind turbines. Advanced wind turbine control systems are an active field of research. Many modern utitility-scale wind turbines feature pitch control and generator torque (or power) control to allow for effective variable-speed operation. In a basic variablespeed wind turbine control system, torque control is used in below-rated wind speeds to achieve maximum energy capture by seeking an optimal tip speed ratio while the blade pitch is held constant. Above rated wind speeds pitch control is utilized to maintain rotor speed while the torque is held constant. ${ }^{1}$

Increasingly however, control is used for objectives additional to speed regulation and power maximization. One common additonal objective is the mitigation of structural loads in the turbine. It has been shown that control systems can provide substantial load reductions in key turbine components and substructures. In the literature, load reductions have been achieved both through torque control and pitch control. Modifications to the torque control have reduced fatique loads on the drivetrain as well as tower side-to-side (SS) bending. Additional pitch control actions have been used to reduce tower fore-aft bending and remove asymmetric loading through independent pitch control. ${ }^{2-4}$ Field demonstration of these load reductions in the literature can be found, for example, in Bossanyi et al or Wright et al. ${ }^{3,4}$

The conventional, or classical, approach to introducing additional damping is by adding separate single-input single-output (SISO) control loops to the existing baseline control loop for each new control objective. In this approach, the SISO loops for each load to be mitigated are designed independentally and then integrated. The resultant controller consisting of several SISO control loops is referred to here as multi-SISO or m-SISO. A process of iteration and tuning follows to ensure the controllers are not coupling harmfully and introducing filters and modifications to individual controllers to provide the best resultant performance. ${ }^{2}$ The m-SISO approach has been used with great success and is largely the approach employed in industry to the best of the authors' knowledge.

Another approach for achieving the multiple objectives of speed/power regulation and load mitigation is to employ a modern multi-input multi-output (MIMO) controller. This approach has also been shown effective for reducing loads. ${ }^{4-7}$ The advantage of the MIMO control design is its ability to design the control loops simultaneously while explicitly accounting for the coupling that occurs between control loops. This ability could be very beneficial as Lazaro et al. demonstrated significant coupling in m-SISO loops on a simulated wind turbine. ${ }^{8}$ Further, with an increasing number of potential control objectives (for example the damping of several lightly damped modes, independent pitch control, active power control) alongside of increasingly large and flexible turbines and potentially offshore and floating mountings, the task of control design becomes more and more complex and the advantages of MIMO design could become more acute. Control design could become even further complicated by the addition of additional sensors (for example LIDAR wind measurements) or actuation, such as structural and mechanical adaptive rotor technology (SMART) concepts with actuation distributed along the blade. ${ }^{9}$ However, the disadvantage of the approach is the apparent heightened complexity as compared to the more intuitive m-SISO approach. ${ }^{7}$

There is literature documenting and demonstrating the ability of both approaches to reduce loads on wind turbines. However, a comparison of each approach in field-testing is needed to fully explore the performance associated with each. At the National Renewable Energy Laboratory's National Wind Technology Center (NWTC), we have begun an ongoing controller comparison study to analyze, through analysis and field-testing, the advantages or disadvanteges of using state-space MIMO controls in place of m-SISO controllers. In this paper, we present results from the initial stages of this comparison study. In this phase, we establish the baseline speed regulating controller and limit the added objectives to two drivetrain coupled modes: drivetrain torsion and tower side-to-side bending. In future phases, we intend to incrementally increase the number of objectives, as well as potentially include additional control designs in the comparison study.

This paper describes the setup of the controller comparison and the design approach taken for all controllers. We also include initial field-testing results. Finally, we present our thoughts on the progress of the comparison study thus far. Section II describes the experimental facility. Section III describes our baseline controller, the m-SISO controller, and the multivariable controller. Section IV provides the experimental results and Section V our conclusions and future steps.

\section{Experimental Setup}

In this section, we describe our experimental facility and our baseline controller. We also define the added control objectives for the m-SISO and the MIMO controller. 


\section{A. CART3 Wind Turbine}

At the NWTC, field-testing has been used for numerous control studies in the past. Two experimental turbines are sited at the NWTC for the purposes of field-testing advanced control systems: the CART2 and CART3 (Controls Advanced Research Turbine 2 or 3-bladed). The 2-bladed CART2 has been used in numerous published controls experiments to date., ${ }^{3,4}$ The CART3 has recently begun operating and performing experiments for advanced controls research and is the turbine we used for this study. ${ }^{9}$ Figure 1 shows the CART3 and Table 1 presents some essential information about the turbine.

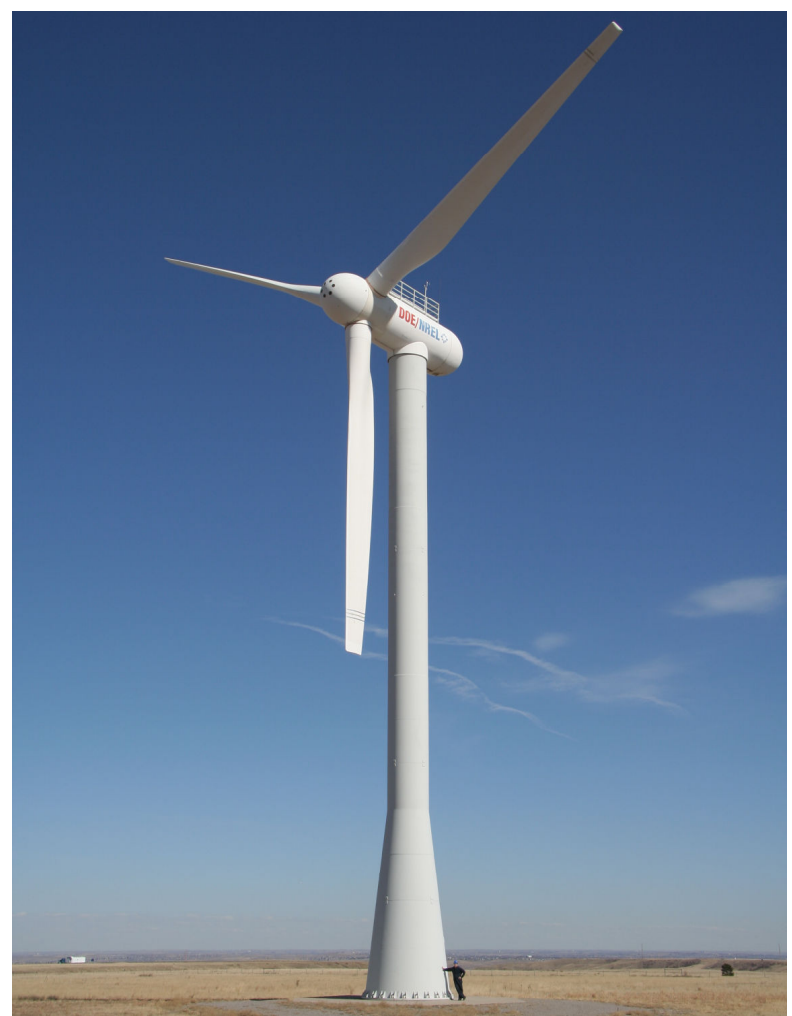

Figure 1: CART3 experimental wind turbine. (NREL/PIX\# 18279)

Table 1: CART3 Turbine Details

\begin{tabular}{|l|l|}
\hline Rated Power & $600 \mathrm{~kW}$ \\
\hline Rotor Diameter & $40 \mathrm{~m}$ \\
\hline Rated Speed & $41.7 \mathrm{RPM}$ \\
\hline Generator Type & Type 4 (Fully de-coupled generator) \\
\hline
\end{tabular}

The CART3 is a $600-\mathrm{kW}$ variable-speed pitch-controlled turbine that currently operates de-rated to $550 \mathrm{~kW} .{ }^{10}$ The CART3 has been modified for use with controls research; it includes an extensive suite of sensors beyond what is typically available in commercial turbines. Additionally, it features a custom control system that facilitates simplified inclusion of an externally designed control system. This research makes extensive use of this capability. In addition to this study, the CART3 is being used in other ongoing controls studies. ${ }^{13}$

\section{B. Controller Comparison}

The main objective of this paper is to compare traditional m-SISO controllers with more advanced MIMO controllers. We are aware that is it rather hard to make a fair comparison of the two controllers and the main conclusion will be that the crucial difference between the two control approaches lies within the tuning process. In 
other words, an experienced engineer can obtain similar results with the two approaches for the control problem that we pose. That is why we give a rather detailed explantion of the tuning process; we design two different controllers and present every step in the tuning process. We add these two controllers on top of a common baseline controller. The baseline controller is designed to be (as much as possible) an industry-standard controller. It features gainscheduled PI (proportional-integral) pitch control for speed regulation in region 3 winds and $\mathrm{k} \omega^{2}$ torque control (which is commanding torque proportional to the square of rotor speed) for maximizing power capture in region 2 winds. This controller follows a standard design approach presented by Jonkman et al. and Hansen et al. ${ }^{11,12}$ Using the same underlying baseline controller for both the m-SISO and MIMO controllers provides a more direct and fair comparison of load reductions. It also allows for load-reducing controllers to be incrementally added to existing wind turbine control loops rather than proposing to replace the whole system.

The baseline controller operates all the time, while the damping controllers are alternatingly ramped on and off. This setup is shown in Figure 2.

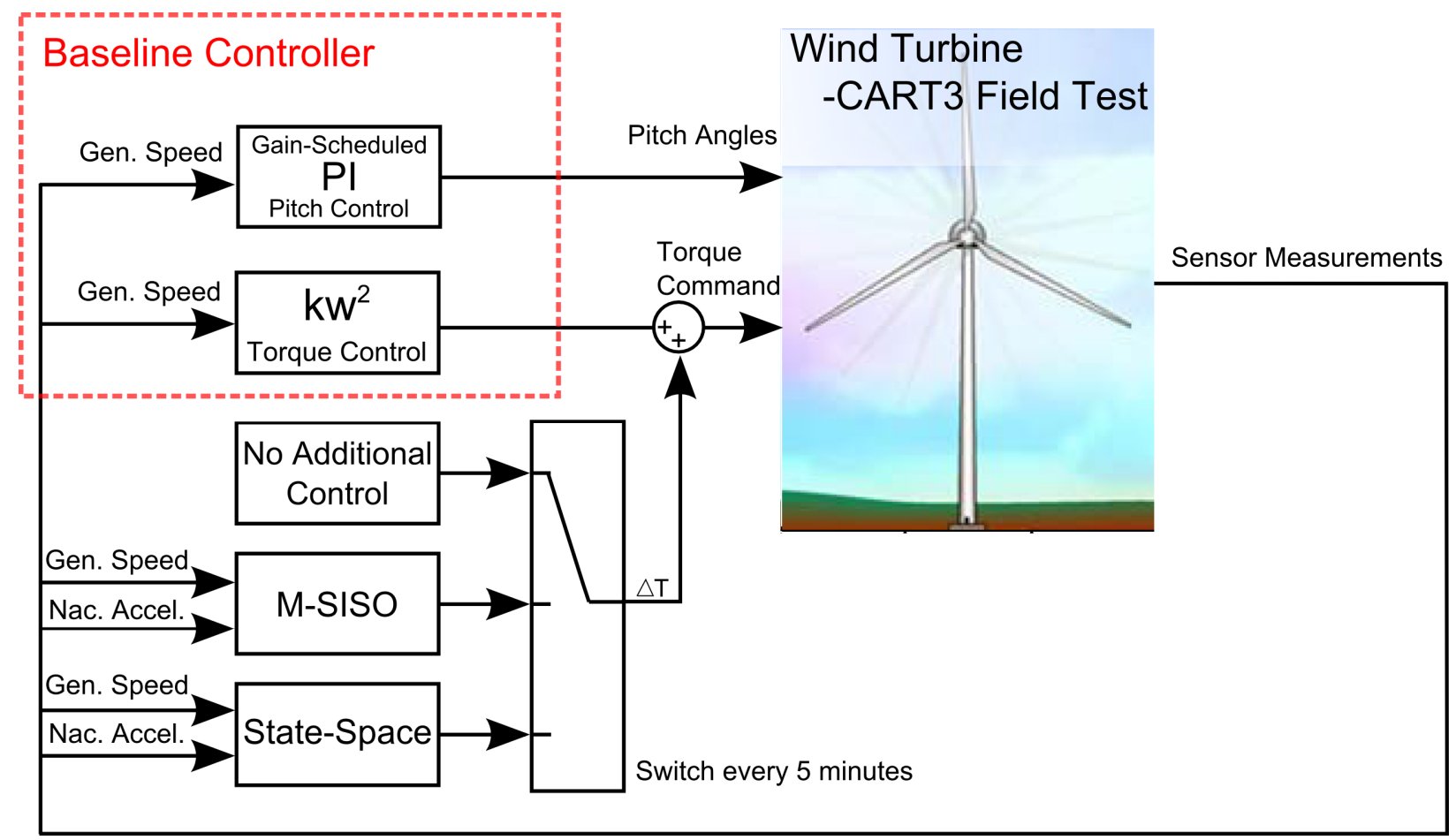

Figure 2. Controller comparison setup

\section{Modes to be damped}

For this first phase of the controller comparison, we construct a test-bench control problem to investigate. The purpose being to develop the test controllers incrementally and methodically. The test-bench control problem uses only a subset of possible control objectives for the full controllers. The main control objective is to minimize the structural loading of the drivetrain and the side-to-side motion of the tower. To be more precise, we would like to add damping to the following modes:

1. The $1^{\text {st }}$ drivetrain torsional mode $(2.7 \mathrm{~Hz})$

2. The $1^{\text {st }}$ tower side-to-side bending mode $(0.88 \mathrm{~Hz})$

A further objective is to minimize the required actuator usage so the controller should only be active at these modes and should not interfere with the baseline controller

Both the tower side-to-side mode and the drivetrain torsion degree-of-freedom can be controlled through modification of the generator torque, so this study is limited to torque-based controls. The sensing used for feedback control is different for the controllers however. Drivetrain torsion will be regulated through feedback of 
the high-speed shaft (HSS) speed while tower bending will be damped through feedback of the nacelles side-to-side acceleration measured by an inertial measurement unit in the nacelle. The two modes can be observed in frequency spectra recorded from the CART3 during operation in Figure 3.

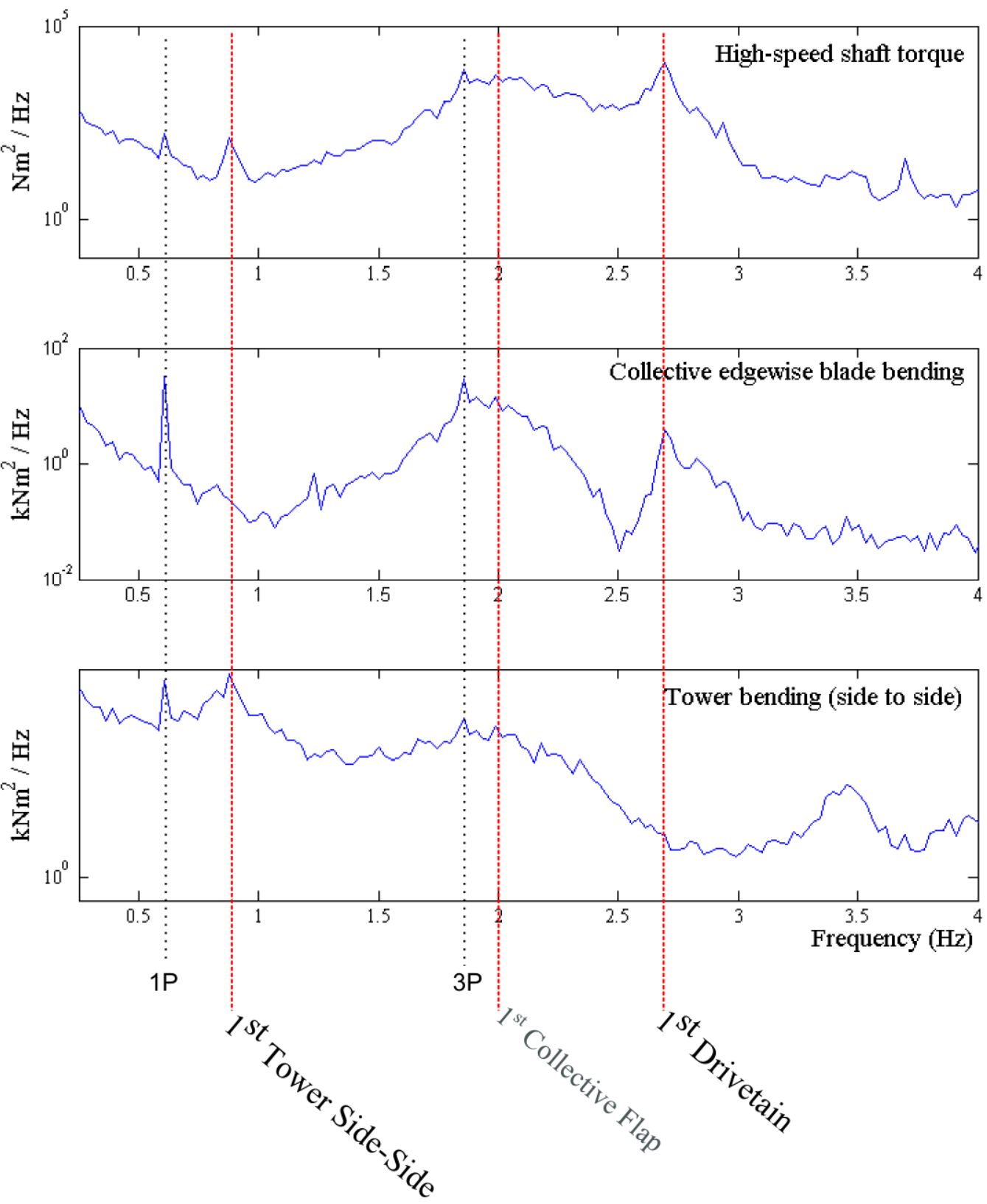

Figure 3. Spectra of signals recorded from CART3 demonstrating modes to be damped, as well as nearby per-rev modes and collective flap.

Figure 3 shows that the two modes to be damped are well-seperated in frequency, making coupling in this case apparently less of an issue. However, challenges to the control design are introduced by the following additional considerations:

- The power electronics determining generator torque has a delay of $50 \mathrm{~ms}$. This is significant, and experience has shown controllers that do not account for this phase loss can destabilize the drivetrain ${ }^{4}$ 
- The measurement of high-speed shaft speed is obtained by differentiation of the shaft encoder and lowpass filtering, leading to additional phase loss and an induced noise signal at $15 \mathrm{~Hz}$ when operating at rated speed

- The power electronics has an apparent intermittent internal oscillation at $4 \mathrm{~Hz}$ and it is important that this frequency not be amplified in feedback ${ }^{10}$

In this section we describe a test-bench field-testing control problem for advanced control design. Although this paper reports on two control designs, we intend to include more designs in the future and refer back to the results obtained by the current controllers. In the next section, we will elaborate further on the design process of the $\mathrm{m}$ SISO and the MIMO controller.

\section{Controller Design}

The design of the two test controllers for comparison will be presented and discussed in this section. This represents one of the more challenging aspects of the study, as we strive for the fairest possible comparison. To achieve this we attempt to use matching components in each design whenever possible. The diagram in Figure 4 attempts to illustrate the design process and the linking of components.

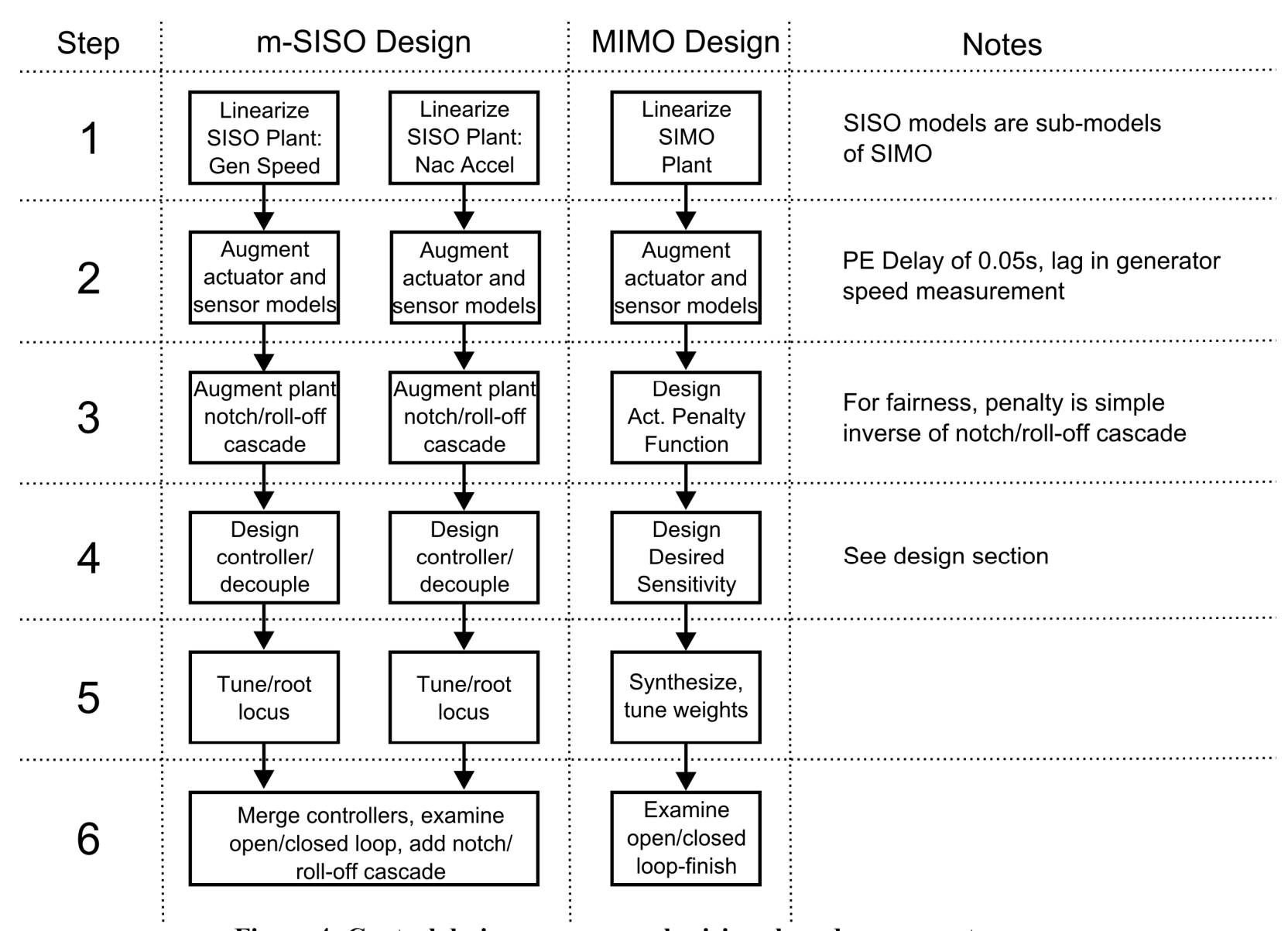

Figure 4: Control design process emphasizing shared components

In the first step in Figure 4, linear models are obtained from a FAST non-linear model of the CART3. The models are identical except that for SISO design, sub-models of the full SIMO (single-input multiple-output) model are obtained. In step 2, the models are augmented with actuator and sensor models, obtained experimentally from the turbine. In step 3, a cascade of notch filters and a roll-off filters is produced to prevent actuator activity at undesirable frequencies (e.g. 1P,2P etc). For the m-SISO case, this is added to the plant to be considered in controller design. For the state-space case, this function is inverted to be used as an actuator penalty function. Steps 
4 and 5 are design methodology dependent, and will be discussed below. Finally, the m-SISO controller is combined into a single controller for implementation on the turbine, and both controllers are examined in open- and closed-loop comparisons.

\section{A. m-SISO design}

As mentioned above, the m-SISO design consists of obtaining linear models for both loops (drivetrain and tower damping), augmenting with sensor and actuator models and then with a cascade of notch and roll-off filters. This cascade prevents actuation at undesirable frequencies. Figure 5 shows a bode plot of the filter transfer function.

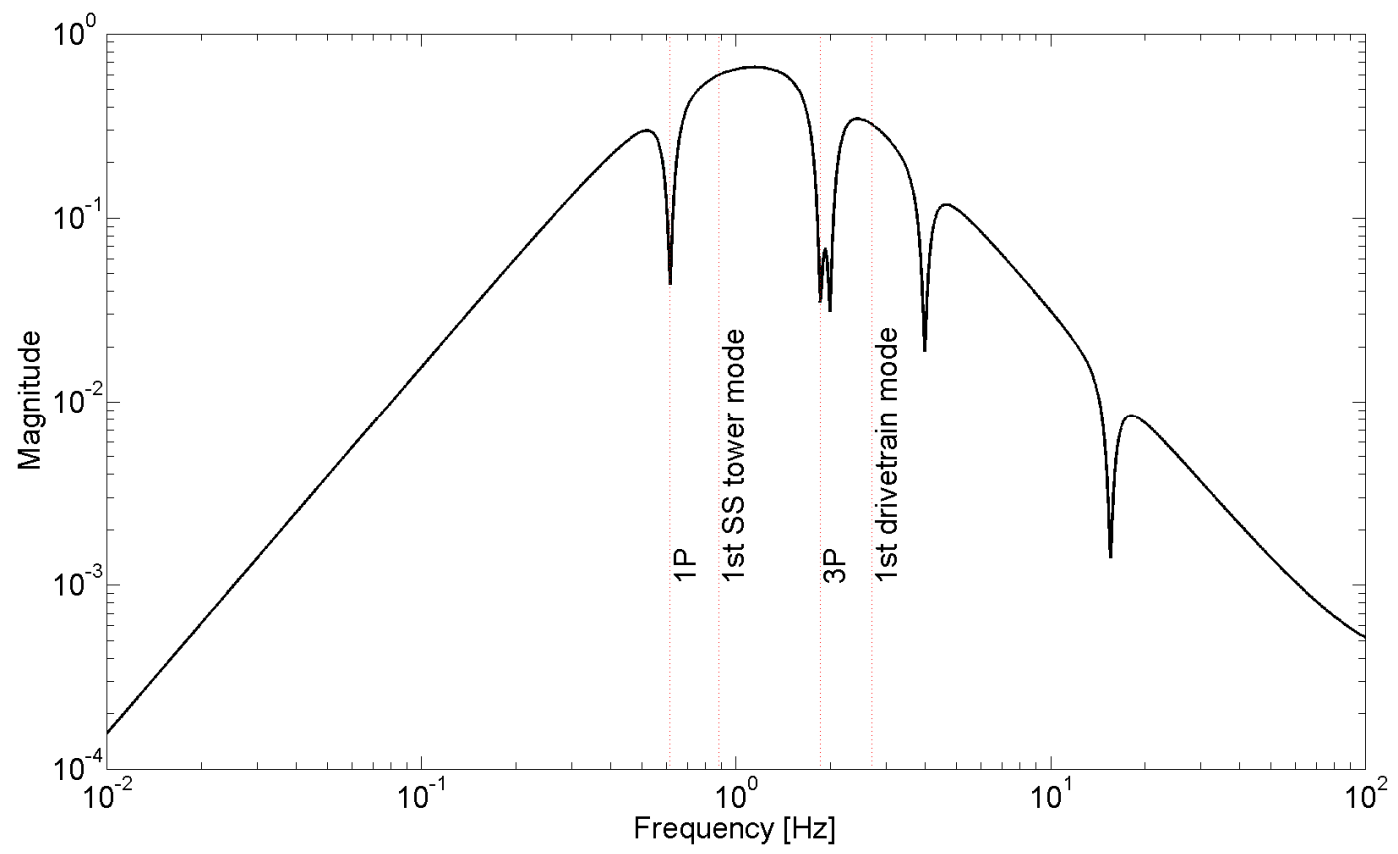

Figure 5: Filter cascade bode magnitude plot

The filter cascade includes roll-off to low and high frequencies, as well as notching at per-rev frequencies, collective flap, power-electronic resonant frequency and speed-sensor noise. This filter function will be added to the controller at the end of the design, but for designing the individual SISO loops, it is appended to the plant.

The next step is to design the two individual loop controllers (drivetrain damping via generator speed feedback and tower side-to-side damping through nacelle acceleration feedback). Both controllers include a notch at the frequency that the other controller is attempting to damp to improve decoupling. The drivetrain controller is composed of a band-pass filter and gain, similar to what is described by Bossanyi. ${ }^{2}$ The gain of the controller was selected by tuning with root-locus. Additionally, the bode plot and gain and phase margins were used to ensure a robust design.

The nacelle controller is composed of an integrator (because the sensed signal is acceleration) and a band-pass filter similar to the one used in the drivetrain damper design. In this case as well, the gain was selected via rootlocus with consideration of open loop bode plot and margins.

Finally, the two controllers were merged yielding a single resultant controller.

\section{B. State-Space design}

For the MIMO state-space design, we used the $\mathrm{H}_{\infty}$ design method, which has a clear frequency domain interpretation. ${ }^{14}$ The key design philosophy here is to design frequency weighting functions that we can apply on the input signal and on our performance signal to shape the frequency response of the closed-loop plant. So, the control objectives defined in the previous sections have to find their way into weighting functions. In particular, we 
would like to penalize both high- and low-frequency control efforts and we only allow for control efforts close to the modes of interest. Furthermore, we prescribe a desired sensitivity function of the closed loop that has notches at the modes we would like to damp.

The weighting function for the actuator penalty function is simply the inverse of the filter cascade used in the mSISO design to limit the number of unnecessary differences between the two design processes. The next step is the design of the output weighting filters, which are in effect the inverse of the desired sensitivity functions for given loops described earlier. The remaining steps are to synthesize the controller and to adjust the gains of the respective weighting functions to achieve a desired damping level on both loops.

\section{Discussion}

A consideration in the selection of control design methodology is the complexity of the design process. One concern of MIMO techniques is the increased complexity of the approach with respect to classical methods. In the case of this study, we noted that the MIMO controller was easier to tune. It took more time and effort to develop a satisfactory m-SISO controller as our initial attempts at decoupling and achieving actuator frequency objectives were not adequate. In fact, it was helpful to compare the bode plot of the m-SISO controller back to the $\mathrm{H}_{\infty}$ during design, suggesting that if state-space controllers are not used as the ultimate controller of a wind turbine, they can be very helpful in directing design to a good controller shape.

In Figure 6, we provide the bode magnitude plots of the two designed controllers.
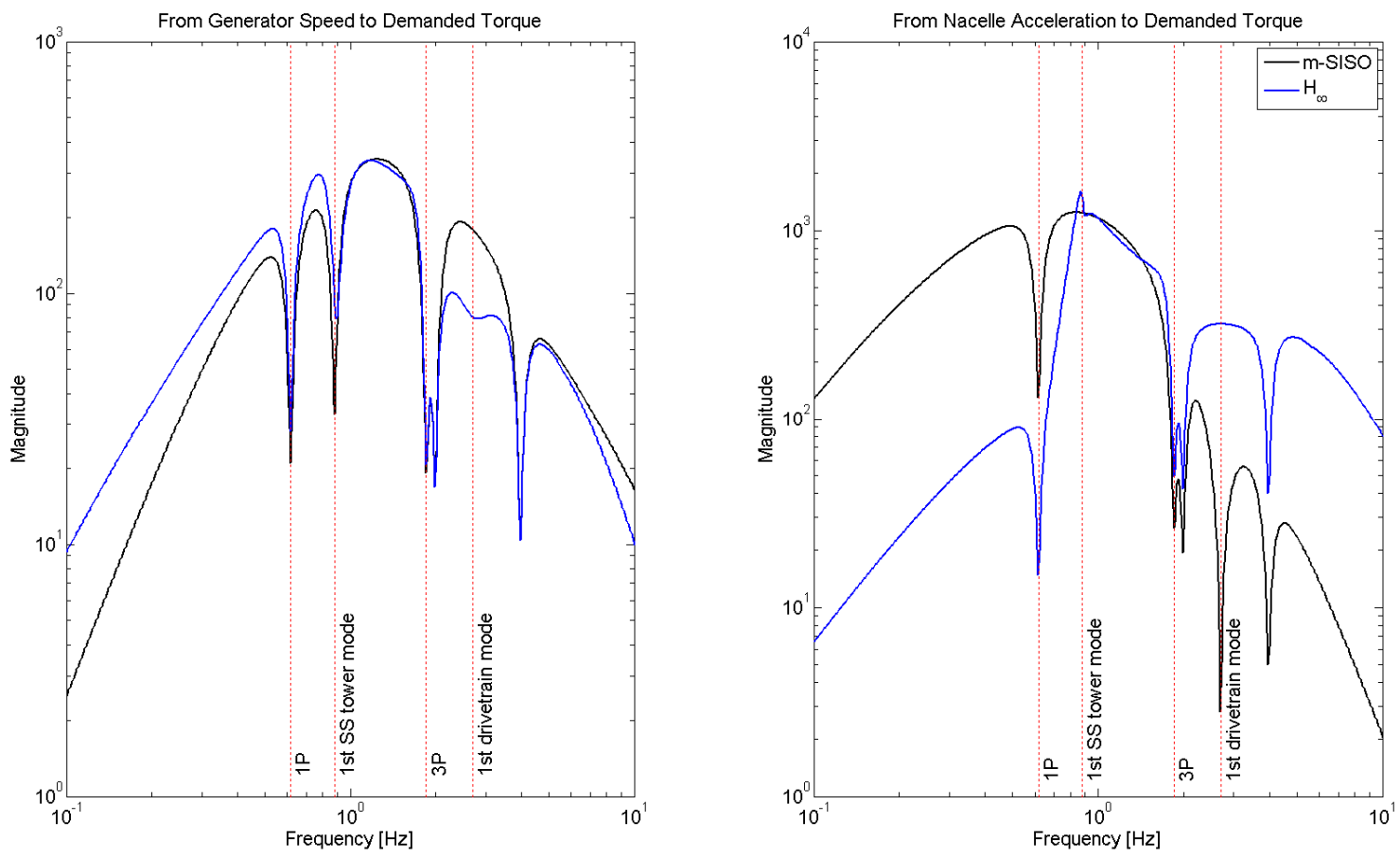

Figure 6: Bode magnitude plot of the MIMO $\mathrm{H}_{\infty}$ controller and the m-SISO controller

Figure 6 shows that the two design processes have yielded somewhat similar controller transfer functions. This is in large part due to the design restrictions on using identical components when possible. However, there are some noticeable differences. One example is the lower gain of the $\mathrm{H}_{\infty}$ controller at the drivetrain frequency in the generator feedback loop and higher gain at the same frequency in the nacelle acceleration feedback loop. 


\section{Field-Testing Results}

Field-testing began once we obtained controllers that performed well in simulation. However, as is often the case, controllers that seemed adequate during the design process, were not adequate when controlling the real turbine, and iterative adjustements had to be made. These adjustments often affected both controllers equally (including an additional notch or adjusting the linear model of the turbine for more accuracy impacts the design of both controllers).

Field-testing of the final controllers is ongoing. Although available data is limited, it is possible to draw inferences from the data collected thus far. A set of 5-minute files with similar statistics was assembled. This seemed to have the most potential as the data sets collected for each controller contain disparate wind scenarios and simply averaging across all runs would be suspect because of the different distribution of wind content of each controller's data sets.

The conformance of the results with expectations given the predicted damping of the controllers raised confidence in the meaningfulness of the results.

\section{A. Field-Testing Comparison}

We identified a set of 5-minute runs with similar conditions for each setup (no damping, m-SISO, MIMO) from the data-set collected. These files and their essential characterisitcs are shown in Table 2.

Table 2: Wind File Set Selected for Comparison

\begin{tabular}{|c|c|c|c|c|c|}
\hline Controller & File Name & $\begin{array}{c}\text { Mean Wind } \\
\text { Speed }\end{array}$ & $\begin{array}{c}\text { Mean Rotor } \\
\text { Speed }\end{array}$ & $\begin{array}{c}\text { Max Rotor } \\
\text { Speed }\end{array}$ & $\begin{array}{c}\text { Min Rotor } \\
\text { Speed }\end{array}$ \\
\hline & & (m/s) & (RPM) & (RPM) & (RPM) \\
\hline Baseline & $\begin{array}{c}\text { CART3 2011 11-28 18-15- } \\
\text { 00.dat }\end{array}$ & 15.945848 & 37.07781 & 37.729126 & 36.532776 \\
\hline M-SISO & $\begin{array}{c}\text { CART3 2011 11-28 18-20- } \\
\text { 0.dat }\end{array}$ & 15.306682 & 37.034974 & 37.836147 & 35.006592 \\
\hline Hinf & $\begin{array}{c}\text { CART3 2011 11-28 18-00- } \\
\text { 00.dat }\end{array}$ & 15.265963 & 37.052347 & 37.749058 & 35.775707 \\
\hline
\end{tabular}

The data in Table 2 shows that all 5-minute runs were collected on the same day. Because the rated wind speed of the CART3 is approximately $12.5 \mathrm{~m} / \mathrm{s}$, all of the runs represent region 3 . The mean wind speeds of the damping controller runs are especially close.

From this data set, we constructed several spectra for comparison. Spectra are created of the two controlled signals (HSS speed and nacelle acceleration) as well as the actuator control signal generator torque command. For each, a power spectral density is computed of the entire 5 minute signal, and then the frequencies are binned for clarity. The result is shown in Figure 7. 

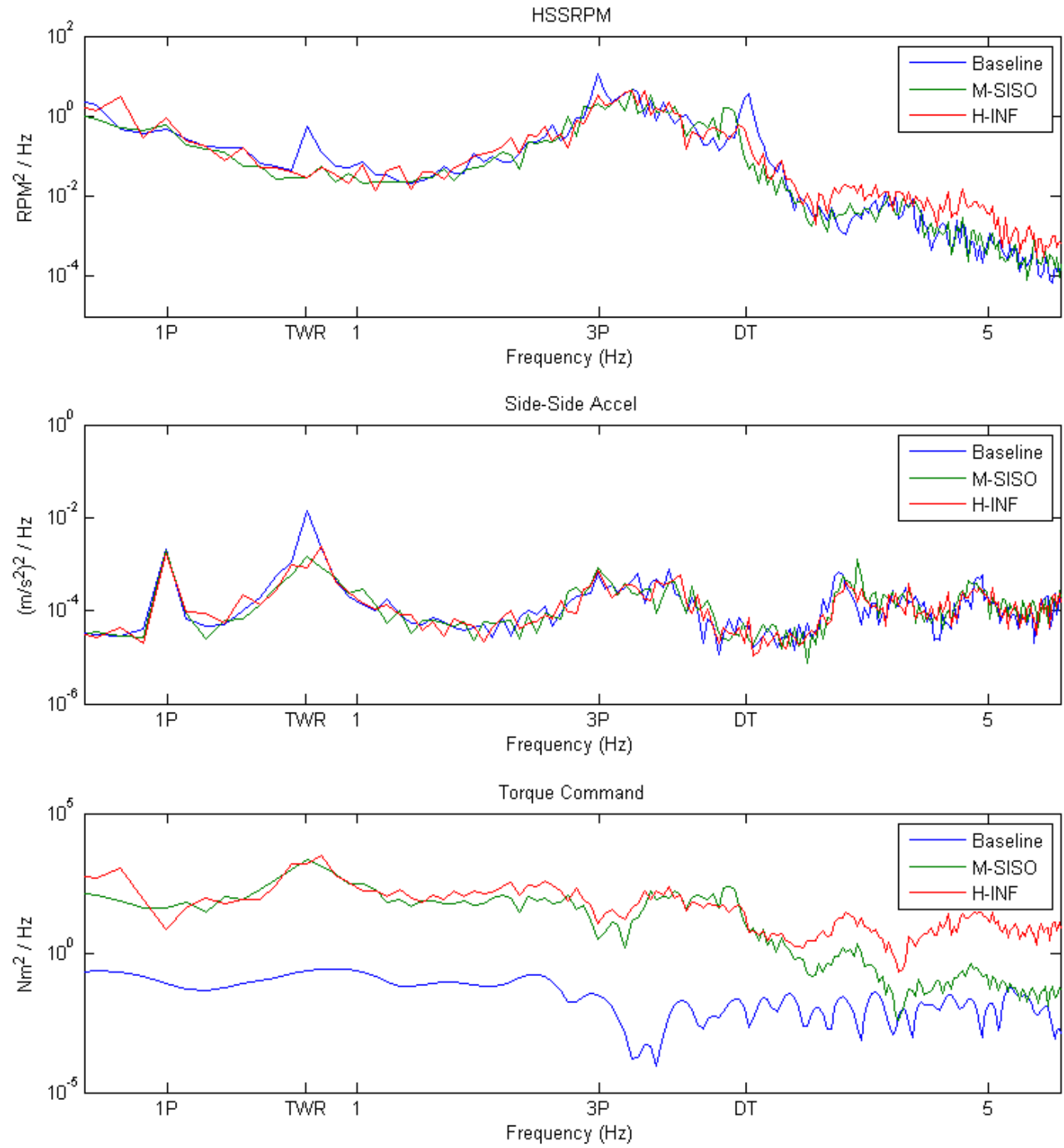

Figure 7: Field-test comparison of controlled and actuation signals

Figure 7 shows that with respect to the HSS RPM signal, the $\mathrm{H}_{\infty}$ controller is the much more effective at damping the drivetrain torsional mode (notice that the m-SISO controller shifts the frequency of the mode to the left). The peak magnitude of the drivetrain frequency for the $\mathrm{H}_{\infty}$ controller is about $29 \%$ of what it is for the mSISO case (which is $45 \%$ of the undamped case). Although the two controllers seem equally effective at damping the tower mode, the m-SISO controller has a slight edge over the $\mathrm{H}_{\infty}$ controller, the significance of which is unclear. Finally, the $\mathrm{H}_{\infty}$ controller has more higher frequency content in the torque actuation signal however, both controllers have met the requirement to avoid excess activity at $1 \mathrm{P}$ and $3 \mathrm{P}$.

In the appendix, we provide similar spectra of all available runs with mean wind speeds above $15 \mathrm{~m} / \mathrm{s}$ in order to indicate that the available data conform with trends observed here. However, the disparity in wind conditions is much greater. 


\section{Discusion and Conclusions}

This paper presents results from the the initial phase of a controller comparison field-test study being conducted at the NWTC. The aim of the study is to investigate the advantages of state-space controller design versus classical approaches. In this first phase, the structure of the comparison is established (with a non-changing baseline controller), and initial controllers are developed for meeting limited objectives. These initial objectives are intended as a test bench and starting point for a continuing comparison study with incremental objectives.

Results available at the time of this writing indicate that, even in the case of only two objectives, there appears to be advanteges to using state-space design methods for the design of controllers for wind turbines. These include explicit accounting of coupling between control loops; an intuitive tuning procedure through frequency weighting functions; and a direct method for expanding the number of sensors, actuators, or control objectives. Further, experience gained in designing the two controllers indicates that including state-space methods in the design process, at least for the purpose of comparison with the deployed controller, is very helpful; comparison with the synthesized multivariable controller helped guide the design of the m-SISO controller The positive results of using state-space controls, even for this simplified problem, is encouraging for the broader study. As more objectives and control loops (as well as possible additional sensing and actuation) are incorporated, the value of using MIMO controllers is expected to increase.

After we have completed the field-tests of the above controllers, we will increase the objectives to include damping via pitch control (tower fore-aft) and independent pitch control. Suggestions from experts on adjustments to make to existing controllers, or controllers to include are welcome. 


\section{Appendix}

In the appendix, we provide the spectra of all data files collected so far in which the wind speed is greater than $15 \mathrm{~m} / \mathrm{s}$ on average.
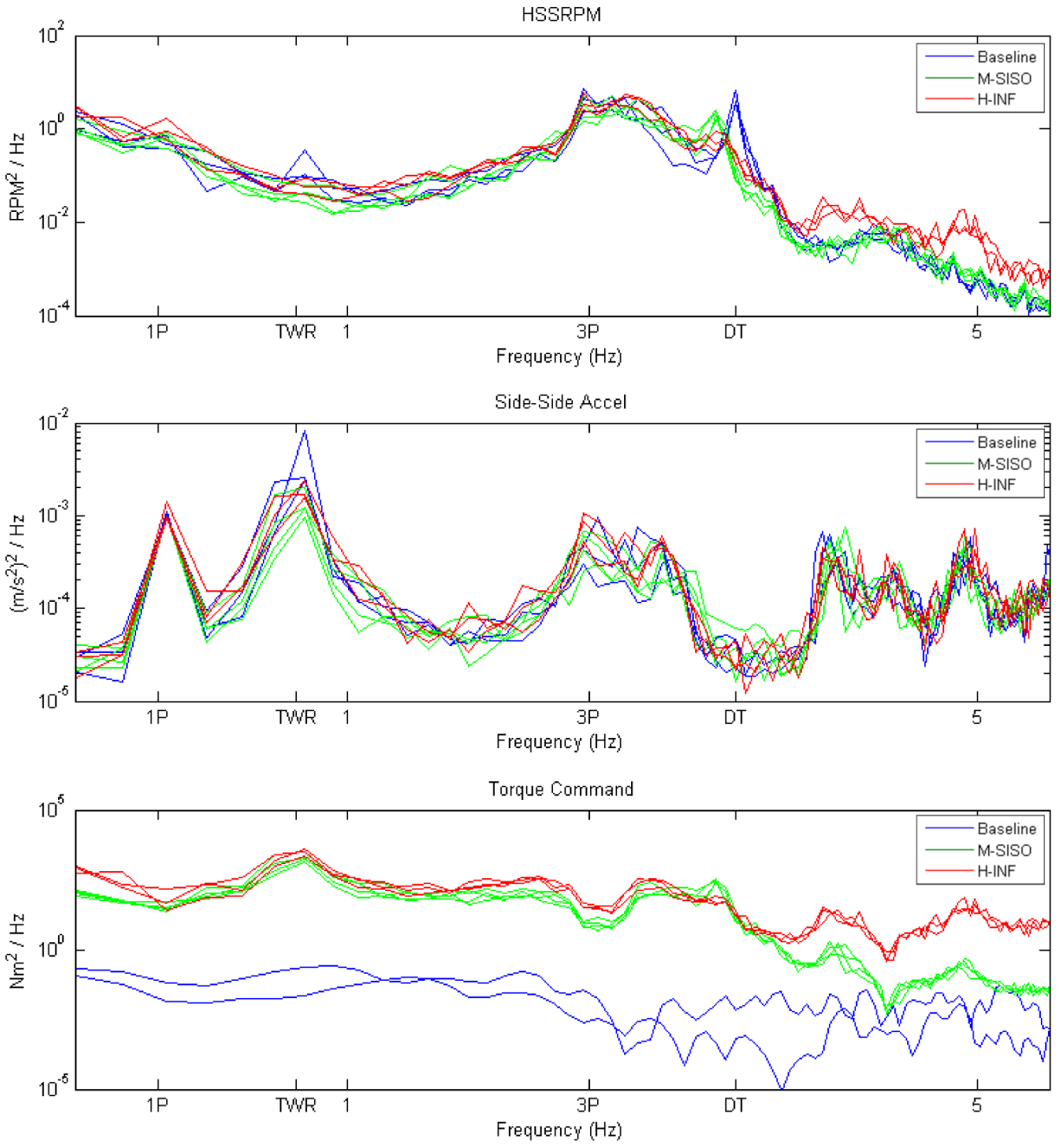

Figure 8. Spectra of signals from all files with mean wind speed greater than $15 \mathrm{~m} / \mathrm{s}$. 
Table 3: Data Sets Used for Appendix Plot

\begin{tabular}{|c|c|c|c|c|c|}
\hline Controller & File Name & $\begin{array}{c}\text { Mean } \\
\text { Wind } \\
\text { Speed }\end{array}$ & $\begin{array}{c}\text { Mean Rotor } \\
\text { Speed }\end{array}$ & $\begin{array}{c}\text { Max Rotor } \\
\text { Speed }\end{array}$ & $\begin{array}{c}\text { Min Rotor } \\
\text { Speed }\end{array}$ \\
\hline Baseline & CART3 2011 11-28 18-15-00.dat & 15.95 & 37.08 & 37.73 & (RPM) \\
\hline Baseline & CART3 2011 11-28 18-30-00.dat & 16.87 & 37.08 & 38.29 & 36.53 \\
\hline Baseline & CART3 2011 11-28 18-35-00.dat & 17.20 & 37.08 & 37.77 & 36.42 \\
\hline M-SISO & CART3 2011 11-28 17-55-00.dat & 17.88 & 37.07 & 37.71 & 36.55 \\
\hline M-SISO & CART3 2011 11-28 18-20-00.dat & 15.31 & 37.03 & 37.84 & 35.01 \\
\hline M-SISO & CART3 2011 11-28 18-40-00.dat & 15.55 & 36.96 & 37.93 & 35.20 \\
\hline M-SISO & CART3 2011 11-28 18-50-00.dat & 15.23 & 37.05 & 38.03 & 35.58 \\
\hline H-INF & CART3 2011 11-28 18-00-00.dat & 15.27 & 37.05 & 37.75 & 35.78 \\
\hline H-INF & CART32011 11-28 18-25-00.dat & 16.65 & 37.07 & 38.06 & 36.37 \\
\hline H-INF & CART3 2011 11-28 18-45-00.dat & 16.27 & 37.07 & 38.16 & 36.30 \\
\hline
\end{tabular}

\section{Acknowledgements}

Invaluable support for this work was provided by Lee Jay Fingersh, Garth Johnson, Scott Wilde, Mark Murphy, Jerry Hur, Syhoune Thao and Don Baker of NREL. NREL's contributions to this report were funded by the Wind and Water Power Program, Office of Energy Efficiency and Renewable Energy of the U.S. Department of Energy under contract No. DE-AC02-05CH11231. The authors are solely responsible for any omission or errors contained herein.

\section{References}

${ }^{1}$ Burton, T., Sharpe, D., Jenkins, N., and Bossanyi, E.A. Wind Energy Handbook, West Sussex, England: Wiley, 2004.

${ }^{2}$ Bossanyi, E.A. "The Design of Closed Loop Controllers for Wind Turbines," Wind Energy, vol. 3, Jul. 2000, p. $149-163$.

${ }^{3}$ Bossanyi, E.A., Wright, A.D., and Fleming, P.A. "Progress with Field Testing of Individual Pitch Control," Conference on the science of making torque from wind, The European Academy of Wind Energy, 2010.

${ }^{4}$ Wright, A.D., Fleming, P.A., and van Wingerden, J.W. "Refinement and Tests of an Advanced Controller to Mitigate Fatigue Loads in the Controls Advanced Research Turbine," 49th AIAA Aerospace Sciences Meeting, 2011.

${ }^{5}$ Stol, K.A., and Fingersh, L.J. Wind Turbine Field Testing of State-Space Control Designs. NREL/SR-500-35061. Golden, CO: National Renewable Energy Laboratory, 2004.

${ }^{6}$ Knudsen, T., Andersen, P., and Toffner-Clausen, S. "Comparing PI and Robust Pitch Controllers on a 400kW Wind Turbine by Full Scale Tests," European Wind Energy Conference, Dublin Castle, Ireland: 1997, pp. 546-550.

${ }^{7}$ Bossanyi, E.A. "Wind Turbine Control for Load Reduction," Wind Energy, vol. 6, Jul. 2003, pp. 229-244.

${ }^{8}$ Lazaro, J.K., Chakiath, M.J., Stol, K.A. and Namik, H. "A Study of Dynamic Coupling and Composite Load Control for Wind Turbines," 48th AIAA Aerospace Sciences Meeting, 2010.

${ }^{9}$ van Wingerden, J.W. et al., "Two-Degree-of-Freedom Active Vibration Control of a Prototyped ' Smart ' Rotor," IEEE Transactions on Control Systems Technology, vol. 19, no. 2, pp. 284-296, 2010.

${ }^{10}$ Fleming, P.A., Wright, A.D., Fingersh, L.J and van Wingerden, J.W. "Resonant Vibrations Resulting from the ReEngineering of a Constant-Speed 2-Bladed Turbine to a Variable-Speed 3-Bladed Turbine," 49th AIAA Aerospace Sciences Meeting, 2011.

${ }^{11}$ Jonkman, J., Butterfield, S., Musial, W., and Scott, G. Definition of a 5-MW Reference Wind Turbine for Offshore System Development Definition of a 5-MW Reference Wind Turbine for Offshore System Development. NREL/TP-500-38060. Golden, CO: National Renewable Energy Laboratory, 2009.

${ }^{12}$ Hansen, M.H., Hansen, A., Larsen, T.J., Øye, S., Sørensen, P., and Fuglsang, P. Control Design for a Pitch-Regulated, Variable Speed WInd Turbine. Risoe-R-1500(EN). Roskilde, Denmark: Risoe National Laboratory, 2005.

13 Bossanyi, E.A., Wright, A.D., and Fleming, P.A. Controller field tests on the NREL CART3 turbine. 11593/BR/09, UPWIND Report, 2011.

${ }^{14}$ Skodestad, S. and Postlethwaite, I. Multivariable feedback control: Analysis and Design, $2^{\text {nd }}$ ed., John Wiley \& Sons, Ltd, 2005.

13

American Institute of Aeronautics and Astronautics 